\title{
To Evaluate the Association between Red Cell Distribution Width and Validated Neurological Scores in Patients with Acute Stroke
}

\author{
J. John Vinoj ${ }^{1}$, Vignesh D. ${ }^{2}$ \\ 1,2 General Medicine, Kanyakumari Government Medical College, \\ Dr MGR Medical University, Tamil Nadu, India.
}

\section{ABSTRACT}

\section{BACKGROUND}

The World Health Organization defined stroke as a "neurological deficit of cerebrovascular cause that persists beyond 24 hours or is interrupted by death within 24 hours". Stroke is one among the leading causes of death and disability in developed as well as developing countries. The red cell distribution width (RDW) is a numeric estimation of the erythrocyte size within the bloodstream. Elevated RDW has been proven to have a predisposing factor in the development of vascular thrombosis and therefore it is an instigator of cerebrovascular diseases. In this study, we intended to evaluate the relationship between RDW values with stroke severity and prognosis in patients in comparison with a healthy control group.

\section{METHODS}

This was an observational cross-sectional study conducted at Kanyakumari Govt Medical College, between June 2021 and December 2021. 150 patients with acute stroke were included in the study along with 150 age-matched controls. Red cell distribution width (RDW) was measured for cases as well as controls. Data were statistically analyzed using GraphPad Prism 8 software.

\section{RESULTS}

Red cell distribution width was positively associated with the occurrence of stroke, particularly ischemic stroke. It was not significantly associated with the occurrence of hemorrhagic stroke.

\section{CONCLUSIONS}

Thus red cell distribution width is a simple, cost-effective, easily attainable novel inflammatory marker that may help in detecting the severity and prognosis of acute ischemic stroke. It can be obtained even at primary health setups and may be used for decision making in the urgent referral of the patient for a better outcome. This study completely supported the routine calculation of this parameter that could add to risk stratification of patients with acute ischemic stroke. It was not significantly associated with the occurrence of hemorrhagic stroke.

\section{KEY WORDS}

RDW, Acute Ischemic Stroke, Cerebrovascular Accident.
Corresponding Author:

Dr. Vignesh D.,

S/o. S. Durairaju,

1/1A Saliyar Street,

Natarajapuram, Musiri-621211,

Trichy District, Tamilnadu, India.

E-mail: vickyd1144197@gmail.com

DOI: $10.14260 /$ jemds/2022/66

How to Cite This Article:

Vinoj JJ, Vignesh D. To evaluate the association between red cell distribution width and validated neurological scores in patients with acute stroke. J Evolution Med Dent Sci 2022;11(02):341-346, DOI: $10.14260 / \mathrm{jemds} / 2022 / 66$

Submission 18-01-2022,

Peer Review 24-01-2022,

Acceptance 02-02-2022,

Published 04-02-2022.

Copyright (C) 2022 J. John Vinoj et al. This is an open access article distributed under Creative Commons Attribution License [Attribution 4.0 International (CC BY 4.0)] 


\section{BACKGROUND}

The World Health Organization defined stroke as a "neurological deficit of cerebrovascular cause that persists beyond 24 hours or is interrupted by death within 24 hours". Stroke is one of the leading causes of death and disability in developed as well as developing countries. Stroke is turning into a crucial explanation for premature death and incapacity in low-income and middle-income countries like the Asian nation, mostly driven by demographic changes and increased by the increasing prevalence of the key modifiable risk factors. The mortality rate of stroke in the acute phase is as high as $20 \%$ and it remains higher for several years after the acute event in stroke patients than in the general population.

In China, the case fatality from hospital-primarily based research is much less than $5 \%$ at 30 days, $10 \%$ at ninety days, and $15 \%$ at 1 year; approximately one 0.33 of sufferers will revel in loss of life or incapacity at three months and 1 year. According to American Heart Association, the mortality rate after stroke ranges from $10.5 \%$ at 30 days, $21.2 \%$ at 1 year, $39.8 \%$ at five years and as much as $58.4 \%$ at the end of life. Despite prognostic factors which include age and the severity of stroke that had been sizeable acknowledged, the prognosis following stroke stays hard to expect due to the complex pathophysiological mechanisms behind the stroke. Given the huge stroke burden, new markers are hard to perceive patients with poor outcomes to be able to appropriately expect a diagnosis and optimize the administration of management.

Cerebral ischemia initiates a complicated cascade of metabolic events, producing nitric oxide and free oxygen radicals. These free radicals and reactive oxygen species (ROS) mediate a brilliant part of injuries appearing after a transitory ischemic attack or in the course of permanent ischemia, modifying macro-molecules, especially DNA, starting up apoptosis and necrosis. Various haematological and biochemical parameters have emerged in recent times which are associated with increased occurrence of stroke. The red cell distribution width (RDW) is a parameter that reflects the heterogeneity of the red blood cell volume. RDW is elevated according to variation in red cell size (anisocytosis), i.e., when elevated RDW is reported on complete blood count, marked anisocytosis (increased variation in red cell size) is expected on peripheral blood smear review. Many researches have suggested that RDW is closely related to many vascular events, such as acute coronary syndrome, ischemic cerebrovascular disease, peripheral artery disease, heart failure (HF), atrial fibrillation (AF) and hypertension. ${ }^{1}$ Red cell distribution width (RDW) is a hematologic parameter that indicates the divergence of red blood cell volume. Increase in RDW has been found in different physiological and pathological conditions such as pregnancy, vitamin B12 and folate deficiency, malignancy, idiopathic pulmonary fibrosis and coronary artery disease. ${ }^{2-7}$ Although RDW was created for the diagnosis of different types of anaemia, recent research has revealed its predictive role in cerebrovascular diseases. ${ }^{8}$ Different studies have shown an increased value of RDW after ischemic stroke. ${ }^{9-13}$ Feng et al. suggested that increased inflammation and oxidative stress during ischemia result in an elevation in RDW and are associated with poor prognosis. However, it is still unclear if RDW can predict the treatment response in stroke patients who are receiving antithrombotic therapy ${ }^{11}$ RDW can be a potential marker to predict the occurrence of stroke in apparently healthy people. Therefore we wanted to evaluate the association of RDW with the occurrence of acute stroke.

\section{METHODS}

This was an observational study conducted from June 2021 to December 2021 in Kanyakumari Govt Medical College hospital, Asaripallam, Tamil nadu. 150 cases of acute stroke primarily diagnosed with a history and clinical examination, and further evaluated by available brain imaging modality (Computerised tomography or MRI) were included in the study. 150 controls were also included who were agematched patients admitted for conditions other than stroke.

\section{Inclusion Criteria}

Patients admitted with symptoms suggestive of acute stroke within 24 hours of the onset of stroke as evidenced by CT scan or MRI.

\section{Exclusion Criteria}

- Patients below 18 years of age.

- Haematological abnormalities like leukaemia or other myeloproliferative disorders.

- Patients of CKD due to any cause

- Haematological conditions related to increased RDW

\section{Data Collection and Methods}

Patients selected after considering inclusion and exclusion criteria underwent detailed history taking followed by clinical examination. This was followed by a haematological and biochemical assessment which included a Complete Blood Count with RDW, and other baseline investigations. A similar assessment was done for individuals included in the control group. Data thus obtained were recorded in a predesigned proforma.

\section{Statistical Analysis}

Data were entered in a Microsoft Excel worksheet. Mean values were calculated for parameters under study. Comparison between mean values was done using an independent sample T-test. P-value $<0.01$ was considered significant. Statistical analysis was done on GraphPad Prism 8 software.

\begin{tabular}{|c|c|c|c|c|}
\hline \multicolumn{5}{|c|}{ RESULTS } \\
\hline Age (Year) & Male & Female & Total & Percent \\
\hline $21-30$ & 2 & 2 & 4 & 2.7 \\
\hline $31-40$ & 9 & 6 & 15 & 10.0 \\
\hline $41-50$ & 22 & 8 & 30 & 20.0 \\
\hline $51-60$ & 24 & 17 & 41 & 27.3 \\
\hline $61-70$ & 21 & 21 & 42 & 28.0 \\
\hline $71-80$ & 6 & 6 & 12 & 8.0 \\
\hline $81-90$ & 1 & 3 & 4 & 2.7 \\
\hline $91-100$ & 1 & 1 & 2 & 1.3 \\
\hline Total & 87 & 63 & 150 & 100.0 \\
\hline
\end{tabular}


As evident from Table 1, among the total of 150 patients studied, the incidence of stroke in the present study was more common in the 5th and 6th decade and there was slight male (58 \%) preponderance compared to females (42\%) making the male-female ratio of 1.4:1 Average age of patients was 55.9 years. Male patients had an average age of 54.5 years while female patients had an average age of 57.7 years.

\begin{tabular}{|c|c|c|c|c|c|c|}
\hline \multirow{2}{*}{$\begin{array}{l}\text { Type of } \\
\text { Stroke }\end{array}$} & \multicolumn{2}{|c|}{ Male } & \multicolumn{2}{|c|}{ Female } & \multirow[b]{2}{*}{ Total } & \multirow[b]{2}{*}{ Percent } \\
\hline & No & $\%$ & No & $\%$ & & \\
\hline Infarct & 54 & 51.9 & 50 & 48.1 & 104 & 69.3 \\
\hline Haemorrhage & 28 & 60.9 & 18 & 39.1 & 46 & 30.7 \\
\hline Total & \multicolumn{2}{|c|}{82} & \multicolumn{2}{|c|}{68} & 150 & 100.0 \\
\hline
\end{tabular}

Table 2 shows the incidence of infarct as (69.3\%) more common compared to haemorrhage (30.7\%). $51.9 \%$ of ischemic stroke occurred in males while $48.1 \%$ occurred in females. $60.9 \%$ of total hemorrhagic stroke occurred in males while $39.1 \%$ occurred in females.

\begin{tabular}{|cccc|}
\hline RDW & Mean (\%) & SD (\%) & \\
\hline Cases ( $=150)$ & 14.42 & 1.64 & P-Value 0.003 \\
Controls ( $\mathrm{n}=150)$ & 13.78 & 1.38 & \\
\hline Table 3. RDW (\%) & in Patients with Stroke Compared with Controls \\
\hline
\end{tabular}

RDW in the stroke patients ranged from $10.09 \%$ to 18.31 $\%$ and in control subjects ranged from $9.86 \%$ to $17.75 \%$. As evident by Table 3, the mean RDW in stroke patients was found to be $14.42 \%$ with a standard deviation of $1.64 \%$, while mean RDW in controls was found to be $13.78 \%$ with a standard deviation of $1.38 \%$. The difference was found to be statistically significant with $\mathrm{P}<0.01$.

\begin{tabular}{|c|c|c|c|}
\hline RDW & Mean (\%) & SD (\%) & \\
\hline Cases (n=104) & 14.58 & 1.77 & P-Value 0.001 \\
\hline Controls $(n=150)$ & 13.78 & 1.38 & \\
\hline
\end{tabular}

As seen in Table 4, RDW in patients with ischemic stroke had a mean value of $14.58 \%$ with a standard deviation of $1.77 \%$ compared with control subjects who had a mean RDW of $13.78 \%$ with a standard deviation of $1.38 \%$. The difference was statistically significant with $\mathrm{P}<0.01$.

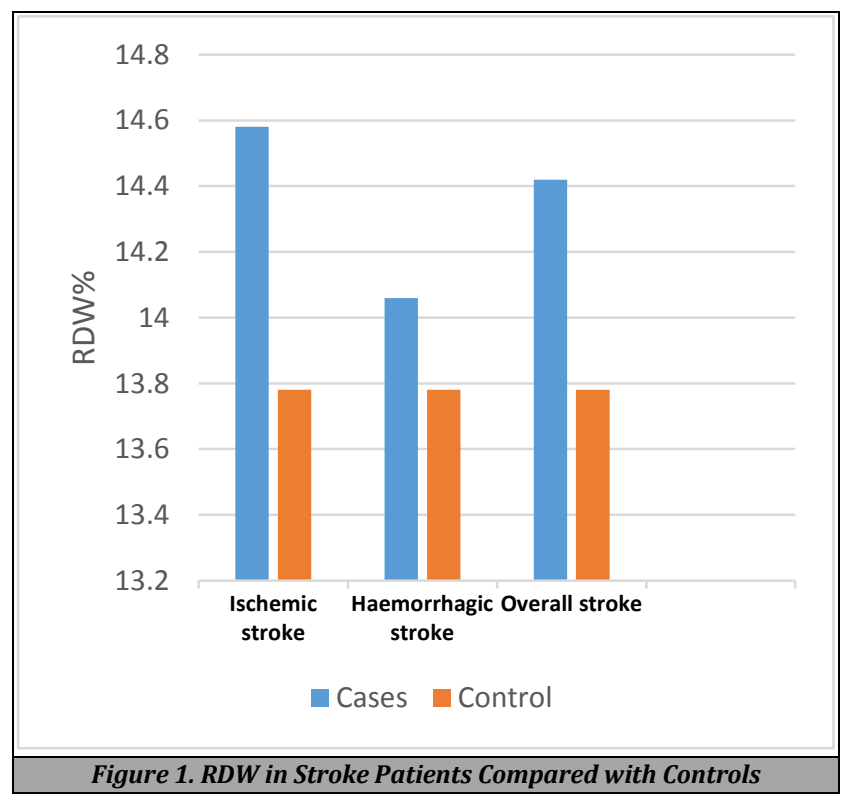

\begin{tabular}{|cccc|}
\hline RDW & Mean (\%) & SD (\%) & P-Value 0.280 \\
\hline Cases ( $\mathrm{n}=46)$ & 14.06 & 1.25 & 1.38 \\
Controls $(\mathrm{n}=150)$ & 13.78 & Controls \\
\hline Table 5. RDW (\%) in Patients with Hemorrhagic Stroke Compared to \\
\hline \multicolumn{4}{|c|}{ Control } \\
\hline
\end{tabular}

As seen in Table 5, RDW in patients with hemorrhagic stroke had a mean value of $14.06 \%$ with a standard deviation of $1.25 \%$ compared with control subjects who had a mean RDW of $13.78 \%$ with a standard deviation of $1.38 \%$. This difference was not found to be statistically significant as the $p$ value was $>0.01$.

\section{DISCUSSION}

Our study showed that mean RDW in stroke patients was higher $(14.42 \pm 1.64 \%)$ compared to controls $(13.78 \pm 1.38$ $\%)$. This difference was statistically significant. A cohort study conducted by Ani et al.(2009) ${ }^{14}$ found that mean RDW was significantly higher among persons with stroke compared to individuals without stroke $(13.7 \%$ vs $13.2 \%$, P $<0.001)$. This was similar to the results obtained by us. A meta-analysis conducted by $\mathrm{Li}$ et al. (2020) ${ }^{15}$ included 6 studies that concluded that RDW level in patients with stroke was significantly higher than in those without stroke $(\mathrm{P}<$ 0.001).

\section{Ischemic Stroke}

In our study, patients with ischemic stroke had a mean RDW of $14.58 \pm 1.77 \%$, which was significantly higher than the RDW of controls which was $13.78 \pm 1.38 \%$ ( $\mathrm{P}<0.01$ ). Our results were comparable to those obtained in a case-control study by Moreno et al. (2013) ${ }^{16}$ where patients with ischemic stroke had a mean RDW of $14.48 \pm 1.76$ compared to controls with mean RDW $13.91 \pm 1.43$. $(\mathrm{P}<0.01)$. A population-based cohort study by Soderholm et al.(2015) ${ }^{17}$ concluded that RDW in the highest quartile was associated with increased incidence of total stroke and cerebral infarction. A metaanalysis by Feng et al. (2017) concluded that increased RDW value was likely associated with ischemic cerebrovascular disease, carotid artery atherosclerosis and cerebral embolism.

\section{Hemorrhagic Stroke}

In our study, mean RDW in patients with hemorrhagic stroke was found to be higher $(14.06 \pm 1.25)$ as compared to controls (RDW $13.78 \pm 1.38$ ). However, this difference was not found to be statistically significant. Soderholm et al. (2015) ${ }^{17}$ in their cohort study concluded that there is no significant association between RDW and incidence of intracerebral or subarachnoid haemorrhage. This is similar to our result. However, a study conducted by Altintas et al. (2017) ${ }^{18}$ showed that there is a significant relationship between RDW and hematoma growth in patients with hypertensive ICH, which indirectly indicates RDW may be associated with the occurrence of hemorrhagic stroke. In a study by Wang et al. (2019) ${ }^{19}$ it was shown that RDW is associated with hemorrhagic transformation in acute ischemic stroke patients, pointing towards a possible association of RDW with ICH. 
Stroke is the result of cerebral vascular occlusion or haemorrhage, and it is the leading cause of death in the world. ${ }^{12-14}$ At present, clinical diagnosis is based on history, neurological examinations and neuro-imaging. Several scoring systems are used to quantify the degree of severity of stroke: the Glasgow Coma Scale, Canadian Neurological Scale, Scandinavian Stroke Scale and National Institutes of Health Stroke Scale (NIHSS). However, there's no biological surrogate marker to diagnose stroke. RDW by using flow cytometry might be a choice for this reason and is expecting the prevalence of stroke.

RDW was a new routine parameter representing the variability in the size of circulating erythrocytes, which could early identify iron deficiency before other tests. Therefore, it could point out the earliest morphologic changes in anaemia caused by deficiency of folic acid, iron, and vitamin B12.20 At the same time, it also happened in some autoimmune diseases, hemolytic anaemia, sickle cell disease, and blood transfusion. ${ }^{21}$ Previous data had reported that higher RDW levels could be an independent predictor of increased stroke incidence, even in those with atrial fibrillation and heart failure. ${ }^{22,23}$ Besides, it was also related to carotid atherosclerosis and hypertension, which could cause some small vascular stroke.24,25 RDW may also be used as a biomarker to predict the functional outcomes and mortality in patients with cerebral infarction. ${ }^{26,27}$ With regard to cryptogenic stroke, some studies had confirmed that RDW $>14 \%$ was an independent predictor to forecast the risk of cryptogenic stroke, which could increase by 2.5 times in patients with RDW $>14 \%{ }^{28}$ However, there were still few studies about RDW and stroke, particularly lacking TIA and hemorrhagic stroke research, all of which needed quite more studies to prove.

The mechanisms between elevated RDW levels and stroke have not yet been fully understood. Inflammatory factors and oxidative stress were considered as 2 different important mechanisms causing cerebral infarction. More evidence about increased inflammatory responses may play an important role and have detrimental effects in the pathophysiology of stroke, both of ischemic and hemorrhagic types. A previous study that suggested elevation of CRP in intracerebral haemorrhage during acute-phase was associated with the outcomes such as death and vascular complications. ${ }^{29}$ Neutrophils induce and activate inflammatory responses, while lymphocytes have antiinflammatory and endothelial protective functions. So this imbalance between them is the basis of inflammatory responses. In general, the elevated NLR (neutrophil to lymphocyte ratio) suggests a strong inflammatory response. Lots of studies have shown that it can be used to predict the prognosis of patients with ischemic and hemorrhagic stroke, which is closely related to worse functional prognosis and increased nosocomial mortality. ${ }^{30-32} \mathrm{RDW}$ may reflect the inflammation state, which leads to impaired erythrocyte maturation and shortened erythrocyte survival. Some researches indicated that inflammatory mediators such as IL6 , TNF- $\alpha$, CRP, and ESR were associated with RDW independent of multiple confounding factors, as a part of the inflammatory process. ${ }^{33,34}$ High oxidative stress and low antioxidant levels may be related to high RDW. ${ }^{35}$ It can damage the erythrocyte membrane and increase the fragility of RBCs. It also reduces the rate of erythroid maturation and
RBCs life span. Besides, higher RDW reflect the agglutination state of RBCs, playing an important role in haemostasis and the fibrinolytic process and leading to vascular occlusion considering it as one of the independent factors. ${ }^{36}$

The relation between high RDW values and chronic systemic diseases has been known for many years. A myriad of studies report increased mortality in acute ischemic stroke with increased RDW. Even though the reason behind the increment in RDW of acute ischemic stroke patients is not completely understood, inflammation is thought to be playing a major role. Inflammation has effects on the red cell production process by disturbing iron metabolism, suppressing erythropoietin at the molecular level and inhibiting the proliferation of erythroid progenitor cells. Stroke severity is usually rated by the NIHSS, which is a reliable predictor of outcome. Recently, several studies have attempted to find a simpler alternative to the NIHSS. Some of these studies have focused on RDW, as it has already been established as a prognostic biomarker in a variety of medical conditions, including cardiovascular disease, lung disease, and diabetes. In a case-control study involving 224 stroke patients and an equal number of matched controls, RDW was found to be a strong predictor of stroke. Additionally, the researchers noted that higher RDW values were associated with an increased risk of stroke. Ani et al. (2009) also found that subjects with RDW values greater than $13.9 \%$ had twice the risk of death as the reference group.

\section{CONCLUSIONS}

Red cell distribution width was positively associated with the occurrence of stroke, particularly ischemic stroke. It was not significantly associated with the occurrence of hemorrhagic stroke. Stroke is one of the leading causes of death and disability worldwide. RDW can help in predicting the occurrence of stroke. Therefore it can work as a tool that can be used to identify individuals at high risk of stroke. Such individuals can be offered lifestyle modification and other interventions which can help in the primary prevention of stroke.

\section{Limitations}

1. This study was done on relatively smaller sample size. A larger sample size would have ensured greater applicability of results to the general population.

2. This was an observational cross-sectional study. A prospective follow up study would have been more helpful in drawing definite conclusions.

3. The RDW has been measured only once and it may change during the patient's hospitalization. Therefore, fluctuations in RDW and changes in RDW over time could not be assessed.

\section{REFERENCES}

[1] Montagnana M, Cervellin G, Meschi T, et al. The role of red blood cell distribution width in cardiovascular and 
thrombotic disorders. Clin Chem Lab Med 2011;50(4):635-41.

[2] Seo SG, Lee MY, Park SH, et al. The association between red cell distribution width and incident hypertension in Korean adults. Hypertens Res 2019;43(1):55-61.

[3] Kar YD, Ozdemir ZC, Emir B, et al. Erythrocyte indices as Differential diagnostic biomarkers of Iron deficiency anemia and thalassemia. J Pediatr Hematol Oncol 2019;42(3):208-13.

[4] Gupta A, Gupta S, Gupta A, et al. Red cell distribution width: a surrogate biomarker to predict tumor burden in carcinoma gallbladder. Niger J Surg Of Publ Niger Surg Res Soc. 2019;25(2):198-202.

[5] Machado IE, Malta DC, Bacal NS, et al. Prevalence of anemia in Brazilian adults and elderly. Rev Bras Epidemiol 2019;22 Suppl 02(Suppl02):E190008.SUPL.2.

[6] Paliogiannis P, Zinellu A, Mangoni AA, et al. Red blood cell distribution width in pregnancy: a systematic review. Biochem Med 2018;28(3):30502.

[7] Zhang Z, Xu X, Ni H, et al. Red cell distribution width is associated with hospital mortality in unselected critically ill patients. J Thorac Dis 2013;5(6):730-6.

[8] Sultana GS, Haque SA, Sultana T, et al. Value of red cell distribution width (RDW) and RBC indices in the detection of iron deficiency anemia. Mymensingh Med J 2013;22(2):370-6.

[9] Kaya A, Isik T, Kaya Y, et al. Relationship between red cell distribution width and stroke in patients with stable chronic heart failure: a propensity score matching analysis. Clin Appl Thromb Hemost 2015;21(2):160-5.

[10] Kara H, Degirmenci S, Bayir A, et al. Red cell distribution width and neurological scoring systems in acute stroke patients. Neuropsychiatr Dis Treat 2015;11:733-9.

[11] Feng GH, Li HP, Li QL, et al. Red blood cell distribution width and ischaemic stroke. Stroke Vasc Neurol 2017;2(3):172-5.

[12] Soderholm M, Borne Y, Hedblad B, et al. Red cell distribution width in relation to incidence of stroke and carotid atherosclerosis: a population-based cohort study. PLoS One 2015;10(5):e0124957.

[13] Vijayashree R, Abirami R, Govindaraju S, et al. Relevance of red cell distribution width (RDW) determination in stroke: a case control study. Int J Sci Res Publ 2014;4(11):1-5.

[14] Ani C, Ovbiagele B. Elevated red blood cell distribution width predicts mortality in persons with known stroke. J Neurol Sci 2009;277(1-2):103-8.

[15] Li B, Liu S, Liu X, et al. Association between red cell distribution width level and risk of stroke: a systematic review and meta-analysis of prospective studies. Medicine (Baltimore) 2020;99(16):e19691.

[16] Ramírez-Moreno JM, Gonzalez-Gomez M, Ollero-Ortiz A, et al. Relation between red blood cell distribution width and ischemic stroke: a case-control study. Int J Stroke 2013;8(6):E36.

[17] Söderholm M, Borné Y, Hedblad B, et al. Red cell distribution width in relation to incidence of stroke and carotid atherosclerosis: a population-based cohort study. PLoS One 2015;10(5):e0124957.

[18] Altintas O, Duruyen H, Baran G, et al. The Relationship of Hematoma Growth to Red Blood Cell Distribution Width in Patients with Hypertensive Intracerebral Hemorrhage. Turk Neurosurg 2017; 27(3):368-73.

[19] Wang C, Wang L, Zhong D, et al. Association between red blood cell distribution width and hemorrhagic transformation in acute ischemic stroke patients. Cerebrovasc Dis 2019;48(3-6):193-9.

[20] Sultana GS, Haque SA, Sultana T, et al. Value of red cell distribution width (RDW) and RBC indices in the detection of iron deficiency anemia. Mymensingh Med J 2013;22(2):370-6.

[21] Sahli CA, Bibi A, Ouali F, et al. Red cell indices: differentiation between beta-thalassemia trait and iron deficiency anemia and application to sickle-cell disease and sickle-cell thalassemia. Clin Chem Lab Med 2013;51(11):2115-24.

[22] Saliba W, Barnett-Griness 0 , Elias $M$, et al. The association between red cell distribution width and stroke in patients with atrial fibrillation. Am J Med 2015;128(2):192e11-8.

[23] Balta S, Demir M, Demirkol S, et al. Red cell distribution width is related to stroke in patients with heart failure. Clin Appl Thromb Hemost 2015;21(2):190.

[24] Finkelstein A, Halkin A, Revivo M, et al. High red blood cell distribution width and preclinical carotid atherosclerosis. Biomarkers 2015;20(6-7):376-81.

[25] Tanindi A, Topal FE, Topal F, et al. Red cell distribution width in patients with prehypertension and hypertension. Blood Press 2012;21(3):177-81.

[26] Pinho J, Marques SA, Freitas E, et al. Red cell distribution width as a predictor of 1-year survival in ischemic stroke patients treated with intravenous thrombolysis. Thromb Res 2018;164:4-8.

[27] Turcato G, Cappellari M, Follador L, et al. Red blood cell distribution width is an independent predictor of outcome in patients undergoing thrombolysis for ischemic stroke. Semin Thromb Hemost 2017;43(1):305.

[28] Kim J, Kim YD, Song TJ, et al. Red blood cell distribution width is associated with poor clinical outcome in acute cerebral infarction. Thromb Haemost 2012;108(2):34956.

[29] Di Napoli M, Slevin M, Popa-Wagner A, et al. Monomeric C-reactive protein and cerebral hemorrhage: from bench to bedside. Front Immunol 2018;9:1921.

[30] Lattanzi S, Brigo F, Trinka E, et al. Neutrophil-tolymphocyte ratio in acute cerebral hemorrhage: a system review. Transl Stroke Res 2019;10(2):137-45.

[31] Lattanzi S, Cagnetti C, Rinaldi C, et al. Neutrophil-tolymphocyte ratio improves outcome prediction of acute intracerebral hemorrhage. J Neurol Sci 2018;387:98-102.

[32] Celikbilek A, Ismailogullari S, Zararsiz G, et al. Neutrophil to lymphocyte ratio predicts poor prognosis in ischemic cerebrovascular disease. J Clin Lab Anal 2014;28(1):2731.

[33] Lippi G, Targher G, Montagnana M, et al. Relation between red blood cell distribution width and inflammatory biomarkers in a large cohort of unselected outpatients. Arch Pathol Lab Med 2009;133(4):628-32.

[34] Forhecz Z, Gombos T, Borgulya G, et al. Red cell distribution width in heart failure: prediction of clinical events and relationship with markers of ineffective 
erythropoiesis, inflammation, renal function, and nutritional state. Am Heart J 2009;158(4):659-66.

[35] Semba RD, Patel KV, Ferrucci L, et al. Serum antioxidants and inflammation predict red cell distribution width in older women: the Women's Health and Aging Study I. Clin Nutr 2010;29(5):600-4.

[36] Wohner N, Sotonyi P, Machovich R, et al. Lytic resistance of fibrin containing red blood cells. Arterioscler Thromb Vasc Biol 2011;31(10):2306-13. 